\title{
Automotive Observers based on Multibody Models and the Extended Kalman Filter
}

\author{
Javier Cuadrado, Daniel Dopico, Miguel A. Naya, Roland Pastorino
}

\author{
Laboratory of Mechanical Engineering \\ University of La Coruña \\ Escuela Politecnica Superior, Mendizabal s/n, 15403 Ferrol, Spain \\ email: javicuad@cdf.udc.es, ddopico@udc.es,minaya@cdf.udc.es, \\ rpastorino@udc.es
}

\begin{abstract}
This work is part of a project aimed to develop automotive real-time observers based on detailed multibody models and the extended Kalman filter (EKF). In previous works, a four-bar mechanism was studied to get insight into the problem. Regarding the formulation of the equations of motion, it was concluded that the state-space reduction method known as matrix-R is the most suitable one for this application. Regarding the sensors, it was shown that better stability, accuracy and efficiency are obtained as the sensored magnitude is a lower derivative and when it is a generalized coordinate of the problem. In the present work, the automotive problem has been already addressed, through the selection of a Volkswagen Passat as a case-study. A model of the car containing fourteen degrees of freedom has been developed. The observer algorithm that combines the equations of motion and the integrator has been reformulated so that duplication of the problem size is avoided, in order to improve efficiency. A maneuver of acceleration from rest and double lane change has been defined, and tests have been run for the "prototype", the "model” and the "observer", all the three computational, with the model having 100 $\mathrm{kg}$ more than the prototype. Results have shown that good convergence is obtained, but the computational cost is high, still far from real-time performance.
\end{abstract}

Keywords: observers, estimators, detailed multibody models, extended Kalman filter, automotive.

\section{INTRODUCTION}

Observers serve to estimate the states of a dynamic system based on a model and on some measurements of the system [1]. In this way, they provide estimates of magnitudes which have not been sensored because it would be either expensive or practically difficult. In many cases, observers must run in realtime, since the information they provide is used to control the actual system.

Usually, the system models are simple, even linear, since their fast evaluation is needed in order to meet the above mentioned real-time requirement [5]. However, detailed models would provide more and more accurate information, thus enabling the implementation of more sophisticated control strategies.

This work is part of a project aimed to develop real-time observers for automobiles based on detailed multibody models and the extended Kalman filter (EKF). However, to get the fundamental insight in the problem, preliminary studies were carried out on a four-bar mechanism with a spring-damper element, as described in the following two paragraphs.

In a first previous work [2], the authors selected the two dynamic formulations which were found to better fit the structure required by the EKF, and compared them: a state-space reduction method known as matrix-R method, and a penalty method. It was concluded that the matrix-R method was faster and more accurate than its counterpart. The penalty method was not able to keep constraint satisfaction, since an increment of the penalty factor increased both the penalty forces which oppose constraint violation and the correction terms coming from the EKF. Therefore, the kinematic position and velocity problems had to be solved at every function evaluation, thus limiting the efficiency of the method and its typical 
advantages: validity of the set of variables during the whole simulation, even in case of changing configurations, and robustness when facing singular positions.

In a second previous work [3], the authors studied the influence of the sensored magnitude in the performance of the observers. In the above-mentioned first work, a position sensor had been considered which measured the value of the generalized coordinate. In the second work, the behavior of the matrix- $\mathrm{R}$ method was analyzed for different sensored magnitudes -position, velocity, acceleration-, and for the cases when the sensored magnitude is or is not a generalized coordinate of the problem. Sensor noise was considered in all the cases. It was found that the observer becomes less stable, accurate and efficient as higher derivatives are measured, thus demanding either to reduce the integration time-step or to increase the value of the measurement noise covariance in order to keep an acceptable behaviour. Moreover, less accurate results were obtained when the sensored variable is not a generalized coordinate of the problem, since the output Jacobian matrix is less exact in that case. Finally, it was shown that integration of the sensor data to simulate the measurement of a lower derivative yields excellent results, likely due to the filtering effect of the integration process.

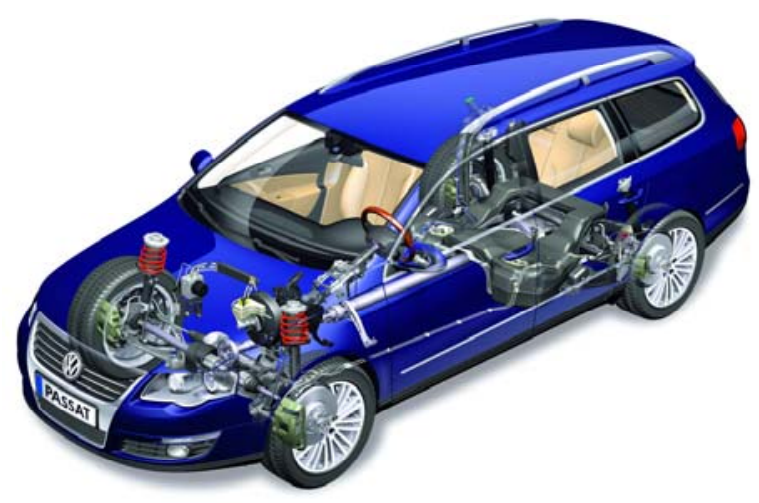

Figure 1. Case-study: Volkswagen Passat.

In the present work, the automotive problem is already addressed. A Volkswagen Passat has been chosen as the case-study. The car has been modeled in natural coordinates from which a subset of fourteen coordinates has been selected to represent the degrees of freedom of the system: chassis translation and rotation, motion of the four suspensions, and wheels rotation. The matrix-R method has been used to write the model equations of motion and, hence, the state-space Jacobian matrix has been recovered from the mentioned previous works. However, looking for better efficiency, the observer algorithm that combines the equations of motion and the integrator has been reformulated, in such a way that duplication of the problem size is avoided, and a nonlinear algebraic system of equations is obtained with the positions as primary variables (velocities are eliminated), thus reducing the problem size to the coordinates number (fourteen in this case, as indicated above); both the residual and the tangent matrix required to apply the Newton-Raphson iterative process have been developed too. The sensored magnitudes considered have been the position level data corresponding to the fourteen dynamic degrees of freedom of the system. A maneuver of acceleration from rest and double lane change has been defined, and tests have been run for the "prototype", the "model” and the "observer", all the three computational, with the model having $100 \mathrm{~kg}$ more than the prototype. Results have shown that good convergence is obtained, but the computational cost is high, still far from real-time performance.

The paper is organized as follows. The second section presents the car chosen as case-study and describes how it has been modeled. The third section shows the observer equations provided by the EKF and pinpoints the two essential elements required to obtain the Kalman gain: the state-space and the output Jacobian matrices. The form of these matrices is provided in the fourth and fifth Sections, respectively. The sixth Section develops the dynamic formulation of the observer, so that duplication of variables is avoided, and the problem is formulated at position level only. The seventh Section explains the simulation which served to test the observer, and shows the results regarding accuracy and efficiency. Finally, the eighth Section gathers the conclusions of the work and indicates the challenges for future research. 


\section{THE CASE-STUDY}

The Volkswagen Passat shown in Figure 1 has been chosen as the case-study. It has been modeled in natural coordinates (44 points and 24 unit vectors), along with some relative coordinates (4 angles and 7 distances), thus leading to a total number of 215 variables. The number of constraints that relate the variables is 208, with some of them redundant, being 15 the number of degrees of freedom: chassis translation and rotation, steering, motion of the four suspensions, and wheels rotation. The front suspension is of McPherson type and requires no special comments. However, the rear multi-link suspension can only work when bushings are placed at the hinges. Since bushings add difficulty to the problem, this first model of the car does not include them and, therefore, the structure of the rear suspension has been slightly modified so that motion is possible without bushings and kinematics remains almost unaltered. The resulting kinematic model is shown in Figure 2.

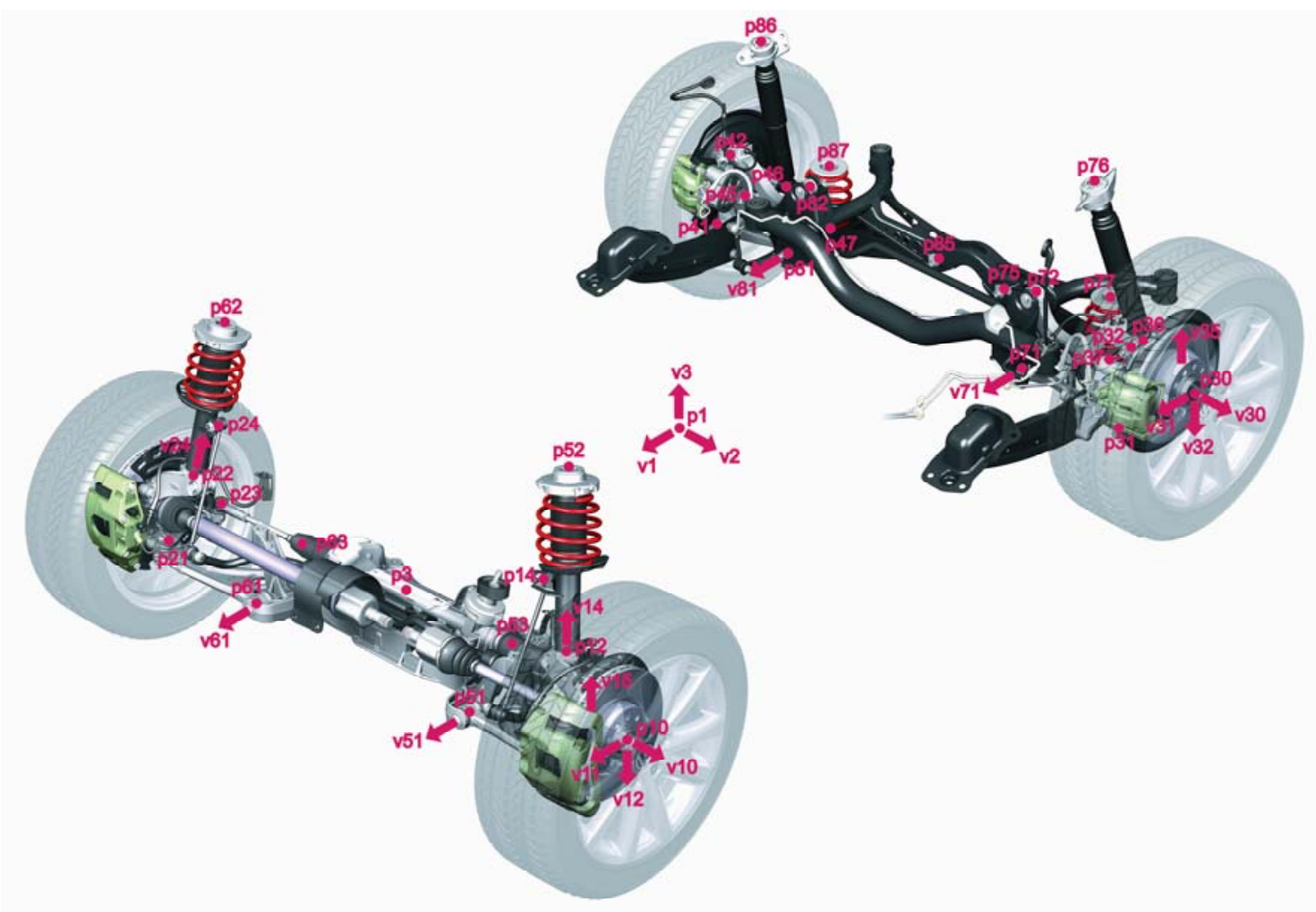

Figure 2. Modeling of the car kinematics.

In what respect to the dynamics, the tires have been considered through a basic linearized formulation with saturation ellipse, the suspensions have been modeled by linear springs and dampers, the anti-roll bars by linear springs too, the steering system has been simulated by kinematic guidance, and the car traction and braking by torques acting on the wheels.

\section{THE EKF EQUATIONS}

A nonlinear system (plant) can be given by the following equations:

$$
\begin{array}{r}
\dot{\hat{\mathbf{x}}}=\mathbf{f}(\hat{\mathbf{x}})+\boldsymbol{\delta} \\
\hat{\mathbf{y}}=\mathbf{h}(\hat{\mathbf{x}})+\boldsymbol{\varepsilon}
\end{array}
$$

where $\hat{\mathbf{x}}$ is the (unknown) state vector, and $\hat{\mathbf{y}}$ is the known measurements vector. The functions $\mathbf{f}$ and $\mathbf{h}$ are also known, and the equations are affected by state and measurement noises $\delta$, $\boldsymbol{\varepsilon}$, with zero mean and given covariances $\Theta, \Xi$, respectively. Then, the EKF is given by [1]: 


$$
\begin{gathered}
\dot{\mathbf{x}}=\mathbf{f}(\mathbf{x})+\mathfrak{Z} \mathbf{k}(\hat{\mathbf{y}}-\mathbf{h}(\mathbf{x})) \\
\mathfrak{z}=\mathbf{P} \mathbb{C}^{\mathrm{T}} \Xi^{-1} \\
\dot{\mathbf{P}}=\mathfrak{A P}+\mathbf{P} \mathfrak{A}^{\mathrm{T}}-\mathbf{P} \mathbb{C}^{\mathrm{T}} \boldsymbol{\Xi}^{-1} \mathfrak{C P}+\boldsymbol{\Theta}
\end{gathered}
$$

being the matrices $\mathfrak{A}$, $\mathbb{C}$, computed as the Jacobians of $\mathbf{f}$ and $\mathbf{h}$ with respect to the states, and evaluated at the estimated trajectory, $\mathbf{x}$. The EKF locally minimizes the covariance $\mathbf{P}$ of the state-estimation error.

Therefore, to build the observer through the EKF technique, two elements are needed: on the one hand, the state-space Jacobian matrix $\mathfrak{A}=\partial \mathbf{f} / \partial \mathbf{x}$ should be obtained, which depends on the form taken by the equations of motion of the model $\dot{\mathbf{x}}=\mathbf{f}(\mathbf{x})$; on the other hand, the output Jacobian matrix $\mathbb{C}=\partial \mathbf{h} / \partial \mathbf{x}$ must be determined, which depends on the sensored magnitudes that will be used to feed the filter.

\section{THE STATE-SPACE JACOBIAN MATRIX}

As said in the Introduction, the matrix-R method [4] has been used to write the model equations of motion which, in turn, serve to obtain the state-space Jacobian matrix $\mathfrak{A}$. The mentioned method is very easily described starting from the classical Lagrange’s equations of the first kind,

$$
\begin{gathered}
\mathbf{M} \ddot{\mathbf{q}}+\boldsymbol{\Phi}_{\mathbf{q}}^{\mathrm{T}} \boldsymbol{\lambda}=\mathbf{Q} \\
\boldsymbol{\Phi}=\mathbf{0}
\end{gathered}
$$

where $\mathbf{M}$ is the positive semidefinite mass matrix, $\ddot{\mathbf{q}}$ the accelerations vector, $\Phi$ the constraints vector, $\boldsymbol{\Phi}_{\mathbf{q}}$ the Jacobian matrix of the constraints, $\boldsymbol{\lambda}$ the Lagrange multipliers vector, and $\mathbf{Q}$ the applied forces vector. Equation (3) represents a system of differential-algebraic equations (DAE).

The matrix-R method obtains a system of ordinary differential equations (ODE) with dimension $n_{i}$ equal to the actual number of degrees of freedom, using a set $\mathbf{z}$ of independent coordinates. To this end, the following relation between velocities is established:

$$
\dot{\mathbf{q}}=\mathbf{R} \dot{\mathbf{z}}
$$

where $\mathbf{q}$ are all the $n_{d}$ dependent variables and $\mathbf{z}$ is a set of $n_{i}$ independent variables. Such a relation can always be found, for instance, taking the derivative of the constraints, $\boldsymbol{\Phi}_{\mathbf{q}} \dot{\mathbf{q}}=\mathbf{0}$, and splitting all the velocities into two subsets, so that one subset of velocities may be written as a function of the other subset. Once Equation (4) has been obtained, it follows that

$$
\ddot{\mathbf{q}}=\mathbf{R} \ddot{\mathbf{z}}+\dot{\mathbf{R}} \dot{\mathbf{z}}
$$

Now, going back to Equation (3), premultiplying by the transpose of $\mathbf{R}$, and having in mind that $\boldsymbol{\Phi}_{\mathbf{q}} \mathbf{R}=\mathbf{0}$,

$$
\ddot{\mathbf{z}}=\left(\mathbf{R}^{\mathrm{T}} \mathbf{M R}\right)^{-1}\left[\mathbf{R}^{\mathrm{T}}(\mathbf{Q}-\mathbf{M} \dot{\mathbf{R}} \dot{\mathbf{z}})\right]=\overline{\mathbf{M}}^{-1} \overline{\mathbf{Q}}
$$

which defines the projected mass matrix $\overline{\mathbf{M}}$ and the projected vector of generalized forces $\overline{\mathbf{Q}}$. The result is that the DAE of Equation (3) in the dependent variables has been converted into the ODE of Equation (6) expressed in the independent variables.

If now the states are defined as $\mathbf{x}^{\mathrm{T}}=\left\{\mathbf{z}^{\mathrm{T}} \mathbf{w}^{\mathrm{T}}\right\}$, with $\mathbf{w}=\dot{\mathbf{z}}$, the following equations can be written,

$$
\begin{gathered}
\dot{\mathbf{z}}=\mathbf{w} \\
\dot{\mathbf{w}}=\left(\mathbf{R}^{\mathrm{T}} \mathbf{M} \mathbf{R}\right)^{-1}\left[\mathbf{R}^{\mathrm{T}}(\mathbf{Q}-\mathbf{M} \dot{\mathbf{R}} \mathbf{w})\right]=\overline{\mathbf{M}}^{-1} \overline{\mathbf{Q}}
\end{gathered}
$$


or, more compactly,

$$
\left\{\begin{array}{c}
\dot{\mathbf{z}} \\
\dot{\mathbf{w}}
\end{array}\right\}=\left\{\begin{array}{c}
\mathbf{w} \\
\overline{\mathbf{M}}^{-1} \overline{\mathbf{Q}}
\end{array}\right\} \Rightarrow \dot{\mathbf{x}}=\mathbf{f}(\mathbf{x})
$$

These equations perfectly match the first set of Equation (1) and, therefore, the state-space Jacobian matrix $\mathfrak{A}$ can be obtained as,

$$
\mathfrak{A}=\frac{\partial \mathbf{f}(\mathbf{x})}{\partial \mathbf{x}}=\left[\begin{array}{cc}
\mathbf{0} & \mathbf{I} \\
\frac{\partial\left(\overline{\mathbf{M}}^{-1} \overline{\mathbf{Q}}\right)}{\partial \mathbf{z}} & \frac{\partial\left(\overline{\mathbf{M}}^{-1} \overline{\mathbf{Q}}\right)}{\partial \mathbf{w}}
\end{array}\right]
$$

which can be approximated as,

$$
\begin{gathered}
\mathfrak{A}=\left[\begin{array}{cc}
\mathbf{0} & \mathbf{I} \\
\mathfrak{A}_{21} & \mathfrak{A}_{22}
\end{array}\right] \\
\mathfrak{A}_{21}=-\overline{\mathbf{M}}^{-1} \mathbf{R}^{\mathrm{T}}\left(\mathbf{K} \mathbf{R}+2 \mathbf{M} \mathbf{R}_{\mathbf{q}} \mathbf{R} \dot{\mathbf{w}}\right) \\
\mathfrak{A}_{22}=-\overline{\mathbf{M}}^{-1} \mathbf{R}^{\mathrm{T}}(\mathbf{C} \mathbf{R}+\mathbf{M} \dot{\mathbf{R}})
\end{gathered}
$$

where $\mathbf{K}$ is the stiffness matrix and $\mathbf{C}$ is the damping matrix.

It should be emphasized that the size of matrix $\mathfrak{A}$ is $2 n_{i}$, i.e. double of the number of degrees of freedom, since the states have been defined as positions and velocities.

\section{THE OUTPUT JACOBIAN MATRIX}

In this first work addressing an automotive example, the sensored magnitudes considered have been the position level data corresponding to the fourteen dynamic degrees of freedom of the system: chassis translation and rotation, motion of the four suspensions, and wheels rotation. Note that the steering has been removed from this list, since it is kinematically guided. More specifically, the sensored variables have been made coincident with the vector of independent coordinates of the system, $\mathbf{z}$, which contains the three coordinates of a point of the chassis to represent its translation (point p1 in Figure 2), three components of unit vectors of the chassis (unit vectors v1, v2 and v3 in Figure 2) that serve to capture its three rotations, the four distances defined at the suspensions, and the four relative angles between hubs and wheels.

Therefore, the output Jacobian matrix $\mathbb{C}$ takes the following trivial form,

$$
\mathfrak{C}=\frac{\partial \mathbf{h}(\mathbf{x})}{\partial \mathbf{x}}=\left[\begin{array}{ll}
\mathbf{I} & \mathbf{0}
\end{array}\right]
$$

with each square submatrix having size $n_{i}$.

\section{THE OBSERVER DYNAMIC EQUATIONS}

In the previous works, the observer equations had been obtained through straightforward combination of the first set of Equation (2) and the integrator equations (trapezoidal rule), since, once the Jacobians $\mathfrak{A}$ and $\mathbb{C}$ are determined, the Kalman gain can be calculated as expressed in the second and third sets of Equation (2). In this way, the resulting observer equations are of size $2 n_{i}$. This fact was not worrying when studying the four-bar mechanism, with one single degree of freedom, but became relevant when addressing the detailed model of the car proposed, with fourteen degrees of freedom. 
Therefore, it was decided to manipulate the equations, so that the final set of observer equations had a size of only $n_{i}$. The starting poin are, on the one hand, the observer equations taken from Equation (2):

$$
\begin{gathered}
\dot{\mathbf{z}}-\mathbf{w}+\mathrm{z}_{1}(\mathbf{y}-\hat{\mathbf{y}})=\mathbf{0} \\
\overline{\mathbf{M}} \dot{\mathbf{w}}-\overline{\mathbf{Q}}+\overline{\mathbf{M}} \mathbf{z}_{2}(\mathbf{y}-\hat{\mathbf{y}})=\mathbf{0}
\end{gathered}
$$

where $\mathbf{z}_{1}$ and $\mathbf{z}_{2}$ are the upper and lower parts of the Kalman gain matrix $\mathbf{z}$, and $\hat{\mathbf{y}}$ are the outputs provided by the sensors. These equations can be rewritten as,

$$
\begin{aligned}
\dot{\mathbf{z}} & =\mathbf{w}-\Delta \mathbf{z}_{1} \\
\overline{\mathbf{M}} \dot{\mathbf{w}} & =\overline{\mathbf{Q}}-\overline{\mathbf{M}} \Delta \mathbf{z}_{2}
\end{aligned}
$$

And, on the other hand, the integrator equations. As mentioned before, the selected integrator has been the implicit, single step, trapezoidal rule.

$$
\begin{aligned}
\dot{\mathbf{z}}_{n+1} & =\frac{2}{\Delta t} \mathbf{z}_{n+1}-\left(\frac{2}{\Delta t} \mathbf{z}_{n}+\dot{\mathbf{z}}_{n}\right) \\
\dot{\mathbf{w}}_{n+1} & =\frac{2}{\Delta t} \mathbf{w}_{n+1}-\left(\frac{2}{\Delta t} \mathbf{w}_{n}+\dot{\mathbf{w}}_{n}\right)
\end{aligned}
$$

where $\Delta t$ is the time-step size. For the sake of simplicity, these equations are rewritten as,

$$
\begin{aligned}
\dot{\mathbf{z}}_{n+1} & =\frac{2}{\Delta t} \mathbf{z}_{n+1}-\hat{\mathbf{z}}_{n} \\
\dot{\mathbf{w}}_{n+1} & =\frac{2}{\Delta t} \mathbf{w}_{n+1}-\hat{\mathbf{\mathbf { w }}}_{n}
\end{aligned}
$$

Specifying the first set of Equation (13) for the time step $n+1$, and using the first set of Equation (15) yields,

$$
\mathbf{w}_{n+1}=\dot{\mathbf{z}}_{n+1}+\Delta \mathbf{z}_{1 n+1}=\frac{2}{\Delta t} \mathbf{z}_{n+1}+\Delta \mathbf{z}_{1 n+1}-\hat{\mathbf{z}}_{n}
$$

which provides the velocities $\mathbf{w}_{n+1}$ as functions of the positions $\mathbf{z}_{n+1}$. This result can be substituted into the second set of Equation (15), leading to,

$$
\dot{\mathbf{w}}_{n+1}=\frac{2}{\Delta t}\left(\frac{2}{\Delta t} \mathbf{z}_{n+1}+\Delta \mathbf{z}_{\mathbf{l}^{n+1}}-\hat{\mathbf{z}}_{n}\right)-\hat{\dot{\mathbf{w}}}_{n}=\frac{4}{\Delta t^{2}} \mathbf{z}_{n+1}+\frac{2}{\Delta t} \Delta \mathbf{z}_{1 n+1}-\overline{\mathbf{w}}_{n}
$$

which provides the accelerations $\dot{\mathbf{w}}_{n+1}$ as functions of the positions $\mathbf{z}_{n+1}$.

Therefore, all the states and derivatives at time step $n+1$ are now expressed as functions of the positions $\mathbf{z}_{n+1}$ through the first set of Equation (15), Equation (16) and Equation (17). If the equations of motion given by the second set of Equation (13) are written for time step $n+1$ in the form of a residual, scaled by a factor of $\Delta t^{2} / 4$, it yields,

$$
\mathbf{g}=\frac{\Delta t^{2}}{4}\left[\overline{\mathbf{M}} \dot{\mathbf{w}}-\overline{\mathbf{Q}}-\overline{\mathbf{M}} \Delta \mathbf{Z}_{2}\right]_{n+1}=\mathbf{0}
$$

Substituting now the value of $\dot{\mathbf{w}}_{n+1}$ provided by Equation (17), the residual $\mathbf{g}$ can be expressed as function of the positions $\mathbf{z}_{n+1}$, leading to the following nonlinear system of equations with the positions $\mathbf{z}_{n+1}$ as unknowns, 


$$
\mathbf{g}\left(\mathbf{z}_{n+1}\right)=\overline{\mathbf{M}} \mathbf{z}_{n+1}-\frac{\Delta t^{2}}{4}\left(\overline{\mathbf{Q}}+\overline{\mathbf{M}} \overline{\mathbf{w}}_{n}\right)+\frac{\Delta t}{2} \overline{\mathbf{M}}\left(\Delta \mathbf{z} \mathbf{z}_{1}+\frac{\Delta t}{2} \Delta \mathbf{z} \mathbf{z}_{2}\right)=\mathbf{0}
$$

This nonlinear system of equations can be solved through the Newton-Raphson iteration, the approximate tangent matrix being,

$$
\begin{gathered}
\frac{\partial \mathbf{g}}{\partial \mathbf{z}}=\overline{\mathbf{M}}+\frac{\Delta t^{2}}{4} \mathbf{R}^{\mathrm{T}}\left[\mathbf{K R}+(\mathbf{C R}+\mathbf{M} \dot{\mathbf{R}})\left(\frac{2}{\Delta t}+\mathbf{z}_{1} \mathbb{C}_{1}\right)\right]+ \\
\frac{\Delta t}{2} \overline{\mathbf{M}}\left[\mathfrak{z}_{1} \mathbb{C}_{1}+\mathfrak{z}_{1} \mathbb{C}_{2}\left(\frac{2}{\Delta t}+\mathbf{z}_{1} \mathbb{C}_{1}\right)+\frac{\Delta t}{2}\left[\mathbf{z}_{2} \mathbb{C}_{1}+\mathbf{z}_{2} \mathbb{C}_{2}\left(\frac{2}{\Delta t}+\mathbf{z}_{1} \mathbb{C}_{1}\right)\right]\right]
\end{gathered}
$$

where $\mathbb{C}_{1}$ and $\mathbb{C}_{2}$ are the upper and lower parts of the output Jacobian matrix $\mathfrak{C}$. Therefore, the dynamic equations of the observer have been reduced to the size of the number of degrees of freedom $n_{i}$, as it was sought.

\section{SIMULATIONS AND RESULTS}

The Volkswagen Passat model described in the second Section has been used here to create three different "cars": a) the "prototype", representing the real system; b) the "model”, representing a non fully accurate computational model of the prototype; c) the "observer", which is nothing but the model corrected with the sensor information coming from the prototype through the EKF.

In the simulated maneuver, the car starts from rest, accelerates until reaching a cruise speed of around 90 $\mathrm{km} / \mathrm{h}$, carries out a double lane change and continues at cruise speed until the end of the simulation time, which is $22.90 \mathrm{~s}$. The time-step size adopted for all the three simulations (prototype, model and observer) was $1 \mathrm{~ms}$. The inaccuracy of the model with respect to the prototype has been introduced by assigning $100 \mathrm{~kg}$ of additional mass to the model. The inputs (steering distance at the rack and traction/braking torque at the rear wheels), identical for both the prototype and the model, are shown in Figure 3.

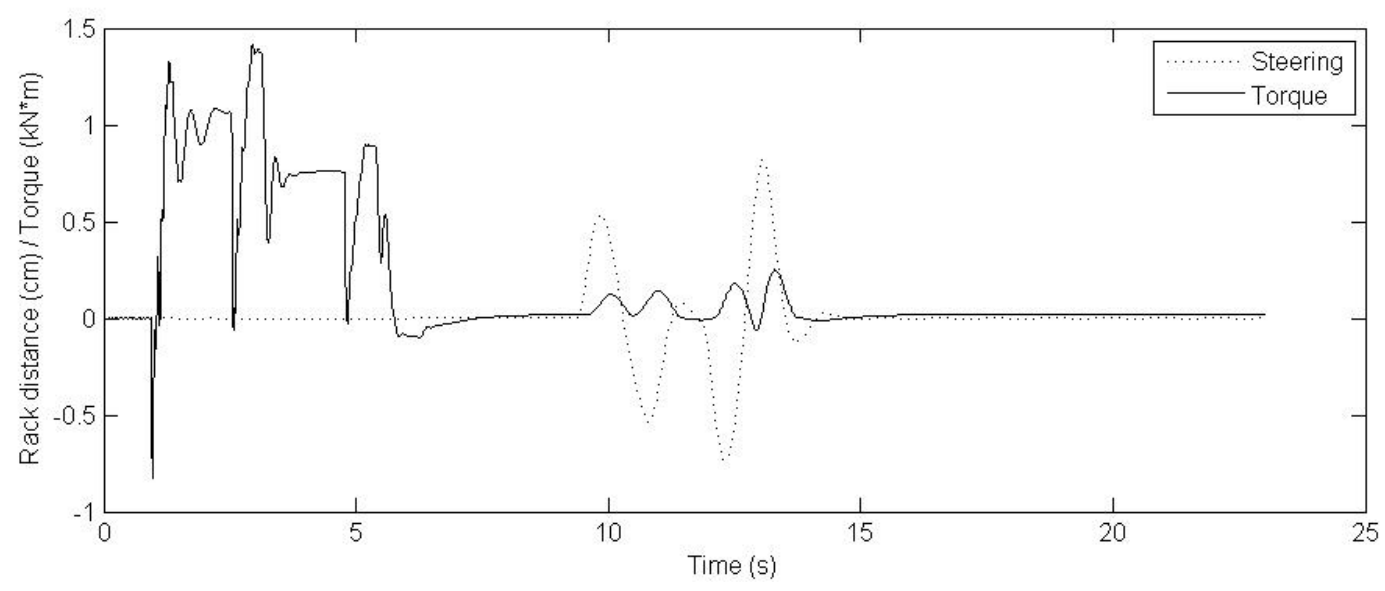

Figure 3. Inputs histories: steering guidance and traction/braking torque.

As said in the fifth Section, the sensored magnitudes considered have been the position level data (free of noise) corresponding to the fourteen dynamic degrees of freedom of the system: chassis translation and rotation, motion of the four suspensions, and wheels rotation. To illustrate the discrepancies between the motion of prototype and model, and the convergence of the observer to the prototype behavior, Figure 4 shows the three coordinates of the chassis center of mass and the three Cardan angles of the chassis for the prototype, the model and the observer. 

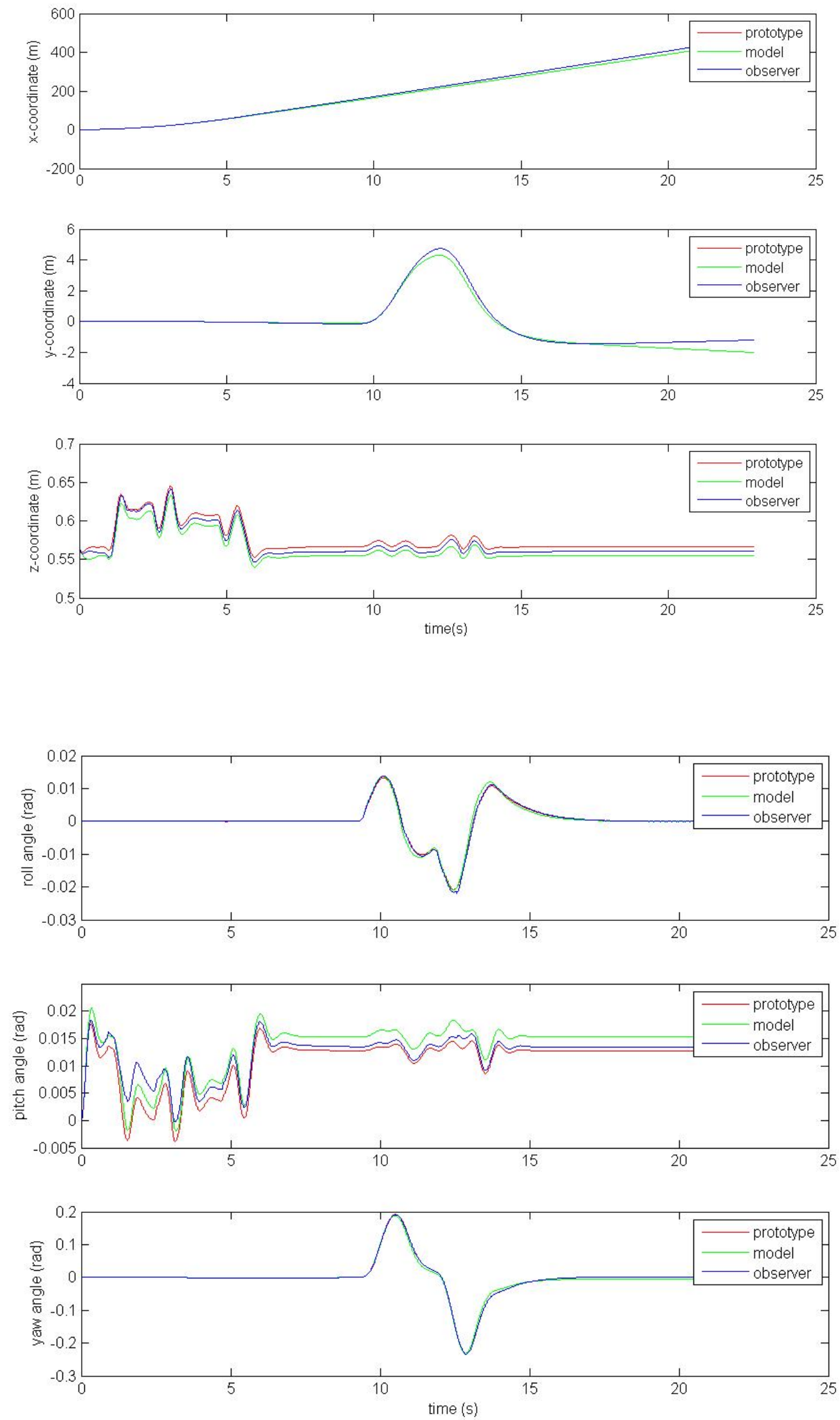

Figure 4. Motion of prototype, model and observer. 
In the figure, it can be seen that the observer achieves good convergence with the prototype. Of course, some magnitudes are not totally preserved, like the vertical coordinate of the chassis center of mass and the chassis pitch angle. These two values are affected by the additional mass of the model with respect to the prototype, which is manisfested through a constant gap in the histories, attenuated in the case of the observer. To reduce this gap, the value of the measurement noise covariance should be increased, at the cost of loosing stability in the observer when sensor noise is considered. Therefore, a trade-off between these two effects should be sought.

\begin{tabular}{|c|c|c|c|}
\hline & Prototype & Model & Observer \\
\hline CPU-time (s) & 48.09 & 48.06 & 228.37 \\
\hline \# of iterations & 22938 & 22935 & 113817 \\
\hline
\end{tabular}

Table 1. Efficiency and NR iterations for prototype, model and observer.

Table 1 shows the total CPU-time spent to run the simulations on a computer Intel Core 2 Duo CPU E6550@2.33 GHz 2.0 Gb RAM, along with the total number of iterations required for the NewtonRaphson procedure. It can be seen that including in the model the EKF corrections to yield the observer makes the code five times less efficient. On the other hand, the average number of iterations required to reach convergence in the Newton-Raphson procedure raises from one in the model to almost five in the observer, which implies a ratio of five times too, meaning that the decrease in efficiency is due to the need of more iterations to attain convergence at each time step, rather than to the dynamic formulation of the observer itself, thus justifying the effort to reduce the size of formulation to the number of degrees of freedom, $n_{i}$.

\section{CONCLUSIONS}

Based on the obtained results, the following conclusions can be drawn:

A Volkswagen Passat observer based on a detailed multibody model of the car and the extended Kalman filter (EKF) has been developed.

To build the observer, the equations of motion have been formulated by means of a state-space reduction method known as matrix-R, so as to yield a set of ordinary differential equations in independent states (positions and velocities), as required by the structure of the EKF, that enables to obtain the state-space Jacobian matrix. To integrate the equations of motion, the implicit, single step, trapezoidal rule has been used. The equations of motion have been combined with the integrator equations in such a way that the size of the problem is kept equal to the number of degrees of freedom of the system, thus avoiding the size duplication imposed by the EKF. This observer dynamic formulation has shown to produce an almost negligible overhead in computational cost with respect to a conventional model, where the Kalman correction is not considered.

The sensored magnitudes considered have been the position level data (free of noise) corresponding to the fourteen dynamic degrees of freedom of the system: chassis translation and rotation, motion of the four suspensions, and wheels rotation.

A maneuver of acceleration from rest and double lane change has been defined, and tests have been run for the "prototype", the "model" and the "observer", all the three computational, with the model having $100 \mathrm{~kg}$ more than the prototype. Results have shown that the observer provides good convergence with the prototype, although a constant discrepancy has been found for some magnitudes, which could be corrected through an increase of the measurement noise covariance. Regarding efficiency, the current code is still far from achieving real-time performance for a constant time-step size of 1 ms, usual in automotive applications related to control, due mainly to the high number of Newton-Raphson iterations required to converge within every time step of integration.

As a consequence of the mentioned conclusions, some challenges are raised for the forthcoming research:

Noise should be introduced in the sensor data, so as to check whether the observer stability is preserved. Furthermore, sensors providing information at velocity and/or acceleration level should be also considered, since they are common in automotive equipment. 
The car modeling and dynamic formulation should be improved to get a more efficient code for the observer, so that real-time performance may be achieved for a time-step size of $1 \mathrm{~ms}$.

\section{ACKNOWLEDGEMENTS}

The support of the Spanish Ministry of Science and Innovation and FEDER through the grant TRA200909314 is greatly acknowledged. Also, the authors are very grateful to the Automotive Technological Center of Galicia (CTAG) and to A. Barreiro from the Department of Systems Engineering and Automatics at Universiy of Vigo, Spain, for their support in this work.

\section{REFERENCES}

[1] Bryson, A. E., AND Ho, Y. C. Applied optimal control. Optimization, estimation and control, New York: Wiley, 1994.

[2] Cuadrado, J., Dopico, D., Barreiro, A., AND Delgado, E. Real-time state observers based on multibody models and the extended Kalman filter. Journal of mechanical science and technology 23, 4 (2009), 894-900.

[3] Cuadrado, J., Dopico, D., Perez, J. A., AND PAStorino, R. Influence of the sensored magntude in the performance of observers based on multibody models and the extended Kalman filter. In Proceedings of the ECCOMAS thematic conference multibody dynamics 2009 (Warsaw, Poland, 29 June - 2 July 2009), K. Arczewski, J. Fraczek and M. Wojtyra, Eds., Warsaw University of Technology, Warsaw, 2009.

[4] GARCIA DE JALON, J., AND BAYO, E. Kinematic and dynamic simulation of multibody systems, New York: Springer-Verlag, 1994.

[5] Wenzel, T. A., Burnham, K. J., Blundell, M. V., AND Willians, R. A. Kalman filter as a virtual sensor: applied to automotive stability systems. Transactions of the institute of measurement and control 29, 2 (2007), 95-115. 